\title{
Experimental Trypanosoma cruzi biclonal infection in Triatoma infestans: detection of distinct clonal genotypes using kinetoplast DNA probes
}

\author{
Artur da Silveira Pinto ${ }^{\mathrm{a}, \mathrm{e}}$, Marta de Lana ${ }^{\mathrm{b}, \mathrm{e}}$, Constança Britto ${ }^{\mathrm{c}}$, \\ Brigitte Bastrenta $^{\mathrm{d}, \mathrm{e}}$, Michel Tibayrenc ${ }^{\mathrm{e}, *}$ \\ ${ }^{a}$ Departamento de Microbiologia, Instituto de Ciências Biológicas, CP 486, Universidade Federal de Minas Gerais, 31270-010, Belo Horizonte, MG, Brazil \\ ${ }^{\mathrm{b}}$ Departamento de Análises Clínicas, Escola de Farmácia, Rua Costa Sena, 171, Universidade Federal de Ouro Preto, Ouro Preto, MG, Brazil, \\ CEP 35.400-000 \\ ${ }^{\mathrm{c}}$ Departamento de Bioquímica e Biologia Molecular, Instituto Oswaldo Cruz, Av. Brasil 4365, Rio de Janeiro, 21045-900, Brazil \\ ${ }^{\mathrm{d}}$ Centre d'Etudes sur le Polymorphisme des Microorganismes (CEPM), Unité Mixte de Recherche no. 9926, Centre National de la Recherche Scientifique \\ (CNRS)/Institut de Recherche pour le Développement (IRD), IRD, Instituto Boliviano de Biologia de Altura, Casilla 9214, La Paz, Bolivia \\ ${ }^{\mathrm{e}}$ Centre d'Etudes sur le Polymorphisme des Microorganismes (CEPM), Unité Mixte de Recherche no. 9926, Centre National de la Recherche Scientifique \\ (CNRS)/Institut de Recherche pour le Développement (IRD), IRD, BP 5045, 34032 Montpellier, Cedex 1, France
}

Received 23 April 1999; received in revised form 27 April 2000; accepted 27 April 2000

\begin{abstract}
Monitored biclonal densities of parasites were offered to third-stage larvae of Triatoma infestans via an artificial feeding device and 30 days later, the gut contents of the insects were processed for microscopic examination and polymerase chain reaction (PCR) detection of Trypanosoma cruzi kinetoplast DNA [kDNA]). A total of 15 mixtures involving nine different stocks attributed to the 19/20, 32 and 39 major clonal genotypes of Trypanosoma cruzi were used. The presence of each T. cruzi clonal genotype after completion of the cycle through the insects was investigated by hybridising the PCR amplification products with genotype-specific minicircle kDNA probes. Sixty-five out of 90 examined insects $(72.2 \%)$ were positive for parasites by microscopic examination and $85(94.4 \%)$ were positive by PCR. The results show that almost half of the biclonal infections are not detectable after completion of the cycle, and that there are important differences in detection of such biclonal infections according to the clonal genotypes considered. Moreover, elimination of a clonal genotype by another is a frequent, but not constant, pattern in biclonal infections of T. infestans. The use of PCR and kDNA probes makes it possible to avoid the culture phase, which makes detection of mixed infections much easier in epidemiological surveys. Moreover, the fact that $T$. infestansdoes not transmit different $T$. cruzi clonal genotypes with the same efficiency has strong implications for the reliability of xenogiagnosis. (C) 2000 Australian Society for Parasitology Inc. Published by Elsevier Science Ltd. All rights reserved.
\end{abstract}

Keywords: Vector; Chagas disease; Transmissibility; Clonal genotype; Clonal competition

\section{Introduction}

Chagas disease remains a major public health problem in Latin America where it has been estimated that 16-18 million people are chronically infected by Trypanosoma cruzi [1]. The parasite is transmitted by triatomine bugs and the widely distributed species Triatoma infestans is considered to be responsible for the contamination of more than one half of infected people (Dujardin J.P., The use of genetic markers in Triatoma infestans and its relevance for vector control, 2nd CDC/ORSTOM/CNRS International Workshop on Molecular Epidemiology and Evolutionary Genetics of Pathogenic Microorganisms,

\footnotetext{
* Corresponding author. Tel.: +33-4-67-41-61-97; fax: +33-4-67-41-62-
}

Montpellier, France, 1997). Population genetic analyses have shown that $T$. cruzi undergoes predominant clonal evolution [2]. Long-term experimental studies by our group have shown that the biological behaviour of $T$. cruzi natural clones is linked to their phylogenetic diversity. Those clones that are phylogenetically distant tend to show very distinct properties for various biological parameters such as doubling time in axenic cultures, in vitro sensitivity to antichagasic drugs, infectivity to cell cultures, pathogenicity in mice and transmissibility through $T$. infestans [3-6]. In the field, it is very frequent to sample isolates from the same host (either man or triatomine bug) that contain two or more distinct clonal genotypes of $T$. cruzi [7-11]. The present work aimed at exploring the dynamics of such mixed infections in laboratory experiments based on artificially-fed $T$. infestans [4,5], and the characterization of 
Table 1

Laboratory code, host and geographic origin of the Trypanosoma cruzi stocks used in the present study

\begin{tabular}{|c|c|c|c|c|}
\hline Clonal Genotype & Stock & Host & Country & Region \\
\hline \multirow[t]{3}{*}{$19 / 20$} & Esquilo cl 1 & Man, acute phase & Venezuela & Cojedes \\
\hline & $\mathrm{SO} 34 \mathrm{cl} 4$ & Triatoma infestans & Bolivia & Potosi \\
\hline & OPS21 cl 11 & Sciurus aestuans ingramini & Brazil & Sao Paulo \\
\hline \multirow[t]{3}{*}{32} & $\mathrm{CBB} \mathrm{cl} 3$ & Man, chronic phase & Chile & Tulahuen \\
\hline & MVB $\mathrm{cl} 8$ & Triatoma infestans & Bolivia & Tupiza \\
\hline & TU18 cl 2 & Man, chronic phase & Chile & Santiago \\
\hline \multirow[t]{3}{*}{39} & $\mathrm{SC} 43 \mathrm{cl} 1$ & Triatoma infestans & Bolivia & Sante Cruz \\
\hline & Bug2148 cl 1 & Triatoma infestans & Brazil & Rio Grand do Sul \\
\hline & $\mathrm{MN} \mathrm{cl} 2$ & Man, chronic phase & Chile & Santiago \\
\hline
\end{tabular}

stocks composed of two distinct clonal genotypes by PCR amplification and use of specific kinetoplast DNA probes $[7,11,12]$.

\section{Materials and Methods}

\subsection{Parasites}

The nine T. cruzi stocks used are listed in Table 1. They were selected from a standardized set of 21 stocks used in our laboratory for all biology studies dealing with $T$. cruzi [3-6]. All stocks have been fully characterized by both multilocus enzyme electrophoresis with 22 different genetic loci [13] and RAPD [14,15]. They have been cloned in the laboratory, with verification under the microscope. Information on the laboratory code, host, and geographic origin of the stocks under survey is given in Table 1. Three main categories of clonal genotypes are represented in the stocks under survey: 19/20, 32 and 39 (after the numbering by [2]). They illustrate different phylogenetic relationships. Indeed, 32 and 39 are more closely related to each other, whereas $19 / 20$ is more distantly related to both 32 and 39 . The nine stocks were previously analyzed for their transmissibility in T. infestans [4]. According to their transmission behaviour in T. infestans, they entered the composition of 15 different mixtures designed to represent various behavioral situations. For each genotype category, either $19 / 20$ or 32 or 39 , we selected three stocks, a 'fast' one (easily transmitted through $T$. infestans), a 'slow' one and a 'medium' one, according to the results obtained by Lana et al. [4]. For each case of biclonal infection $(19 / 20+32,19 / 20+39$, $32+39$ ), five different classes were designed, namely medium/medium, fast/fast, slow/slow, slow/fast and fast/ slow, resulting in a total of 15 mixtures (Table 2). This protocol aims at eliminating a 'stock effect', that is to say: the differences observed are not due to the fact that the stocks under study pertain to a given clonal genotype, but rather, to the individual biological characteristics of these stocks. With this approach, meaningful results are obtained for mixtures of clonal genotypes rather than for mixtures of particular stocks.

\subsection{Experimental conditions}

Experiments were undertaken with $T$. infestans $\mathrm{L} 3$ reared in laboratory conditions, i.e. at $26-27^{\circ} \mathrm{C}, 65-70 \%$ of relative humidity and allowed to feed on chickens every 3 weeks. The insects originated from an outbred colony with individuals coming from Chile, Uruguay and Brazil. They were exposed to infection by using middle-log phase cultures forms from LIT medium, at $28^{\circ} \mathrm{C}$ in an artificial xenodiagnosis device through latex membrane. Eight milliliters of citrated mouse blood, containing exactly one half of each $T$. cruzi clonal genotype assayed at the final concentration of $5.0 \times 10^{5}$ parasite cells $/ \mathrm{ml}$, was used. The system was maintained at $37^{\circ} \mathrm{C}$ and continuously mixed with a magnetic stirrer. The insects were allowed to feed for $30 \mathrm{~min}$ and only the engorged individuals were retained for further analysis. Parasite-free experimental protocols were used as negative controls. Thirty days later, the whole digestive tube of each insect was removed and gently ground in $0.5 \mathrm{ml}$

Table 2

Mixtures of stocks representative of the 19/20, 32 and 39 Trypanosoma cruzi clonal genotypes used to infect third stage larvae of Triatoma infestans. Medium, fast, slow $=$ behaviour in uniclonal infections

\begin{tabular}{|c|c|c|c|c|c|}
\hline \multirow[t]{2}{*}{ Genotype mixture } & \multicolumn{5}{|l|}{ Stock mixture } \\
\hline & Medium + medium & Fast + fast & Slow + slow & Slow + fast & Fast + slow \\
\hline $19 / 20+32$ & Esquilo cl1 + CBB cl3 & SO34 cl1 + MVB cl8 & OPS $21 \mathrm{cl} 11+$ TU18 $\mathrm{cl} 2$ & OPS21 cl11 + MVB cl8 & $\mathrm{SO} 34 \mathrm{cl} 4+\mathrm{TU} 18 \mathrm{cl} 2$ \\
\hline $19 / 20+39$ & Esquilo cl $1+\mathrm{MN} \mathrm{cl} 2$ & $\mathrm{SO} 34 \mathrm{cl1}+\mathrm{SC} 43 \mathrm{cl} 1$ & OPS21 cl11 + Bug2148 cl1 & OPS 21 cl11 + SC43 cl1 & SO34 c14 + Bug2148 cl1 \\
\hline $32+39$ & $\mathrm{CBB} \mathrm{cl} 3+\mathrm{MN} \mathrm{cl} 2$ & MVB cl8 + SC43 cl1 & TU18 cl $2+$ Bug2148 cl1 & TU18 cl2 + SC43 cl1 & MVB cl8 + Bug2148 cl1 \\
\hline
\end{tabular}


Eppendorf tubes containing $10 \mu \mathrm{l}$ of phosphate buffered saline (PBS), pH 7.2. The suspension was then homogenized and $5 \mu \mathrm{l}$ was used to prepare fresh slide smears covered with $22 \times 22 \mathrm{~mm}$ lids. Two hundred microscope fields of each preparation were examined for the presence of parasites, with magnification of $200 \times$.

\subsection{Polymerase chain reaction $(P C R)$}

DNA extraction was performed as described in [16,17]. A $7 \mu \mathrm{l}$ aliquot of the resuspended DNA was amplified by PCR using $T$. cruzi-specific minicircle primers S35 (5'AAATAATGTACGG(T/G)GAGATGCATGA-3 ${ }^{\prime}$ ) and S36 (5'-GGGTTCGATTGGGGTT GGTGT-3') [18]. The primers were annealed to the four conserved regions of each $T$. cruzi minicircle molecule [19,20]. PCR conditions have been described previously [16,19]. Denaturation, annealing, and elongation steps were performed for a total of 35 cycles, for $1 \mathrm{~min}$ each at 94,60 , and $72^{\circ} \mathrm{C}$, respectively. A 330 bp PCR amplification product which encompassed all of the adjacent variable regions and a small portion of the conserved region (approximately $70 \mathrm{bp}$ ) was generated. The amplified products were electrophoresed in a $0.8 \%$ agarose gel and visualized by ethidium bromide staining. Samples from uninfected insects and
DNA-free (negative template control) were also included in each PCR run.

\subsection{Probes, labeling and hybridisation}

Three probes specific for $T$. cruzi clonal genotypes [7,12] were used. The PCR-amplified $270 \mathrm{bp} \mathrm{HVRm} \mathrm{fragment} \mathrm{was}$ purified by electrophoresis on $0.8 \%$ preparative low melting point ultrapure agarose gels (BRL). The fragments were eluted from the agarose using glass beads according to the instructions of the manufacturer (Bio 101), and then digested with the restriction endonucleases Sau96 I and ScaI (BRL). After digestion, the DNA was precipitated with ethanol and resuspended in $100 \mu$ l of distilled water. Oligonucleotide probes were labeled by ${ }^{32} \mathrm{P}$. The PCR electrophoresed samples were transferred onto charged Hybond $\mathrm{N}+$ nylon membranes (Amersham) by vacuum blotting. Each membrane included a negative control (uninfected insects) and three PCR positive controls corresponding to the three categories of clonal genotypes 19/20, 32 and 39 .

Membranes were hybridized at $27^{\circ} \mathrm{C}$ in $6 \times \mathrm{SSC}(1 \times \mathrm{SSC}$ is $0.15 \mathrm{M} \mathrm{NaCl}$ plus $0.015 \mathrm{M}$ sodium citrate)-0.1 SDS1 $\times$ Denhart's solution-0.05 Na Ppi, washed in $6 \times \mathrm{SSC}-0.1 \%$ SDS at $37^{\circ} \mathrm{C}$ for $20 \mathrm{~min}$, and then washed under high stringency conditions in $3 \mathrm{M}$ tetramethyl ammonium chloride at

Table 3

Detection of Trypanosoma cruzi 19/20, 32 and 39 clonal genotypes in experimentally-infected Triatoma infestans

\begin{tabular}{|c|c|c|c|c|c|c|c|}
\hline \multirow{2}{*}{$\begin{array}{l}\text { Genotype and stock } \\
\text { mixture }\end{array}$} & \multirow{2}{*}{$\begin{array}{l}\text { Number of } \\
\text { examined insects }\end{array}$} & \multirow{2}{*}{$\begin{array}{l}\text { Positive } \\
\text { microscopic } \\
\text { examination }\end{array}$} & \multirow{2}{*}{$\begin{array}{l}\text { Positive } \\
\text { HVRm-PCR } \\
\text { amplification }\end{array}$} & \multicolumn{4}{|c|}{ Specific probe recognition } \\
\hline & & & & $\begin{array}{l}\text { First } \\
\text { genotype } \\
\text { only }\end{array}$ & $\begin{array}{l}\text { Second } \\
\text { genotype only }\end{array}$ & $\begin{array}{l}\text { Neither first nor } \\
\text { second genotype }\end{array}$ & $\begin{array}{l}\text { Both } \\
\text { genotypes }\end{array}$ \\
\hline \multicolumn{8}{|l|}{$19 / 20+32$} \\
\hline Medium + medium & 6 & 5 & 6 & 1 & 0 & 0 & 5 \\
\hline Fast + fast & 6 & 6 & 6 & 6 & 0 & 0 & 0 \\
\hline Slow + slow & 6 & 3 & 5 & 0 & 5 & 1 & 0 \\
\hline Slow + fast & 6 & 5 & 6 & 0 & 6 & 0 & 0 \\
\hline Fast + slow & 6 & 6 & 6 & 6 & 0 & 0 & 0 \\
\hline Total & 30 & $25(0.83)$ & $29(0.96)$ & $13(0.43)$ & $11(0.36)$ & $1(0.03)$ & $5(0.16)$ \\
\hline \multicolumn{8}{|l|}{$19 / 20+39$} \\
\hline Medium + medium & 6 & 5 & 6 & 0 & 0 & 0 & 6 \\
\hline Fast + fast & 6 & 2 & 6 & 4 & 0 & 0 & 2 \\
\hline Slow + slow & 6 & 5 & 5 & 0 & 5 & 1 & 0 \\
\hline Slow + fast & 6 & 4 & 4 & 1 & 0 & 2 & 3 \\
\hline Fast + slow & 6 & 6 & 6 & 1 & 0 & 0 & 5 \\
\hline Total & 30 & $22(0.73)$ & $28(0.93)$ & $6(0.20)$ & $6(0.20)$ & $3(0.10)$ & $16(0.53)$ \\
\hline \multicolumn{8}{|l|}{$32+39$} \\
\hline Medium + medium & 6 & 4 & 6 & 0 & 3 & 0 & 3 \\
\hline Fast + fast & 6 & 2 & 5 & 0 & 0 & 1 & 5 \\
\hline Slow + slow & 6 & 3 & 5 & 0 & 0 & 1 & 5 \\
\hline Slow + fast & 6 & 3 & 6 & 0 & 0 & 0 & 6 \\
\hline Fast + slow & 6 & 6 & 6 & 0 & 0 & 0 & 6 \\
\hline Total & 30 & $18(0.60)$ & $28(0.93)$ & $0(0.0)$ & $3(0.10)$ & $2(0.06)$ & $25(0.83)$ \\
\hline Total general & 90 & $65(0.72)$ & $85(0.94)$ & $19(0.21)$ & $20(0.22)$ & $6(0.06)$ & $46(0.51)$ \\
\hline
\end{tabular}


$65^{\circ} \mathrm{C}$ for $30 \mathrm{~min}[16]$. Detection of the ${ }^{32} \mathrm{P}$-labeled probe was performed by exposure of the blot to X-ray film at $-80^{\circ} \mathrm{C}$.

\section{Results}

Table 3 shows the numerical results obtained in the present study. Some typical results are presented on Fig.1. As expected, PCR amplification was more efficient for parasite detection in triatomine bugs than the direct microscopic examination (94.4 and $72.2 \%$, respectively). This rate of successful amplification was roughly the same whatever the pattern of mixture considered. Despite the heavy exposure to infection permitted by the present protocol, we observed some insects in which the presence of parasites was not detectable by PCR (see Tables 2 and 3). The overall rate of successful detection of the two genotypes after comple- tion of the cycle was $51.1 \%$. This rate varied greatly according to the mixture considered: $16.6 \%$ for $19 / 20+32,53.3 \%$ for $19 / 20+39,83.3 \%$ for $32+39$. These differences were statistically significant, as verified by $\chi^{2}$ analysis (19/ $20+32$ vs. $19 / 20+39: 10^{-2}>P>10^{-3} ; 19 / 20+32$ vs. $32+39$ : $\quad P<10^{-3} ; 19 / 20+39 \quad$ vs. $\quad 32+39$ : $2.5 \times 10^{-2}>P>10^{-2}$.

\section{Discussion}

PCR amplification is significantly more sensitive than microscopic examination for the detection of parasites after completion of the cycle, as verified by $\chi^{2}$ analysis $(P>0.001)$. In our results, the overall rate of successful detection of biclonal infection after completion of the cycle was only $51.1 \%$. In a previous study [5], the combined use of
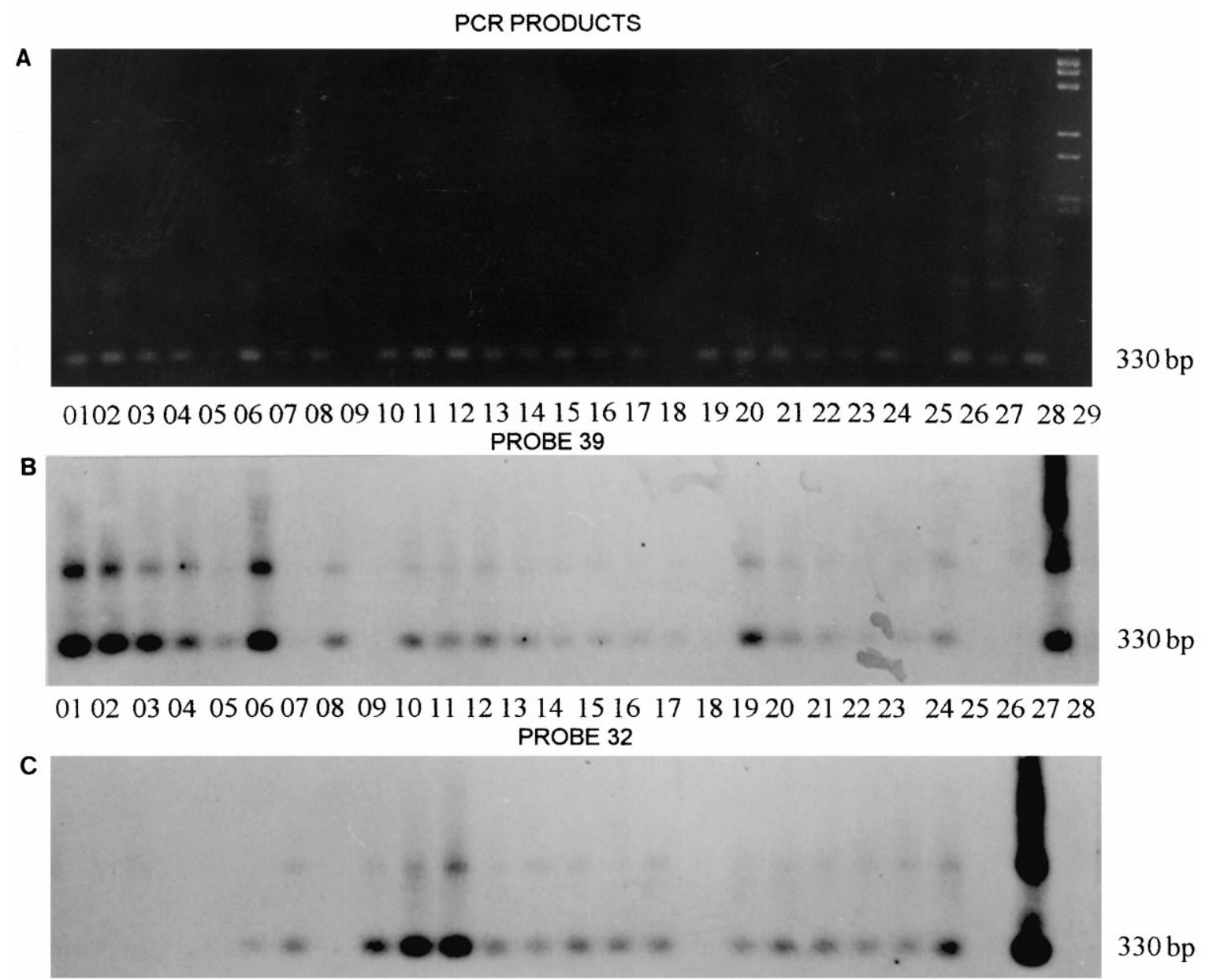

330 bp

01020304050607080910111213141516171819202122232425262728

Fig. 1. PCR amplification and hybridisation with kDNA probes specific for given Trypanosoma cruzi clonal genotypes of various biclonal infections. Each set of six lanes corresponds to six different triatomine bugs infected with the same biclonal infection. Lanes 1 to 6: biclonal infection CBB cl3 (medium genotype 32) + MN cl2 (medium genotype 39); lanes 7 to 12: biclonal infection MVB cl8 (fast genotype 32) + SC43 cl1 (fast genotype 39): lanes 13 to 18: biclonal infection TU18 cl2 (slow genotype 32) + bug2148 cl1 (slow genotype 39); lanes 19 to 24: biclonal infection MVB cl8 (fast genotype 32 ) + bug 2148 cl1 (slow genotype 39); lane 25: negative control with unifected triatomine bug; lane 26: control probe 32; lane 27: control probe 39; lane 28: control probe 19/20. Medium, fast, slow refer to the stock behaviour previously estimated in uniclonal infections (Lana, et al., 1998). A = total amplification; B $=$ hybridization with probe $39 ; \mathrm{C}=$ hybridisation with probe 32 . 
MLEE and RAPD did much better, with a success rate of $87 \%$. The difference is highly significant, as verified by $\chi^{2}$ analysis $(P<0.001)$. This difference is probably due to the fact that both MLEE and RAPD rely on cultivation, and the amount of parasite cells available for detection is therefore much greater than that for the PCR amplification performed in the present study.

The present results clearly show that the composition of biclonal mixtures is not neutral for the successful identification of the two clonal genotypes after completion of the cycle. A genotype effect is apparent, as verified by the analysis above. The same tendency was raised in a previous study from our group [5] with the combined use of MLEE + RAPD, but the differences in that research were not statistically significant.

Moreover, for a given design of biclonal mixture (either $19 / 20+32$ or $19 / 20+39$ or $32+39$ ), a stock effect may be suspected, but the small sample sizes make any statistical verification difficult. In the case of the 19/20 + 32 mixture, the only successfully identified mixture after completion of the cycle was with the medium/medium stocks. All other mixtures could not be detected. Yeates' corrected $\chi^{2}$ is highly significant $(P>0.001)$. In the other mixtures, the stock effect is much less apparent.

It is worth noting that although a different efficiency of transmission through $T$. infestans was noted between $T$. cruzi clonal genotypes (by decreasing order: 19/20, 39, 32 ; [4]), in the present study, for any of the biclonal mixture patterns, there is no obvious tendency of one genotype to eliminate the other. This was already noted by looking for the persistence of mixtures with MLEE and RAPD [5]. This suggests that the behaviour of biclonal infections is different from a simple juxtaposition of the behaviour of the clonal genotypes they are composed of, and could explain the persistence in nature of many different clonal genotypes exhibiting a high diversity of biological parameters.

Moreover, when the problem of xenodiagnosis is considered, in confirmation with previous studies from our group [4,5], the present results show that the genotype composition of the stocks is definitely not neutral for the efficiency of parasite detection, since here, an obvious genotype effect could be detected.

Natural mixtures of $T$. cruzi clonal genotypes are very frequent [11]. We have proposed [20] that the biological relevance of these mixtures could be high (phenomena of clonal cooperation and clonal hitch-hiking). The methodological approach presented here, based on PCR detection of mixtures, although it is less sensitive than the combined use of MLEE and RAPD, is much more operational for extensive epidemiological studies aiming at evaluating the frequency of mixed infections in various ecological cycles and their impact on the transmission and pathogenicity of Chagas disease. As a matter of fact, the PCR approach avoids the tedious step of parasite culturing, which is required for MLEE and RAPD analysis.

\section{Acknowledgements}

We are most grateful to Dr G.A. Schaub, Ruhr-Universität Bochum, Germany, Prof D. Le Ray (Institute of Tropical Medicine, Antwerp, Belgium) and Prof J. Jurberg. (Instituto Oswaldo Cruz, Rio de Janeiro, Brazil), who kindly provided the Triatoma infestans adult individuals that made it possible to establish our $T$. infestans rearing. This work was supported by the WHO Special Program for Research and Training in Tropical Diseases ( $\mathrm{n}^{\circ}$ 910268), an EEC STD3 grant no. TS3* CT92-0155, and a Groupement de Recherche grant CNRS/French Army. Dr Artur. da S. Pinto, Dra. Marta de Lana were supported by fellowships from Conselho Nacional do Desenvolvimento Científico e Tecnológico (CNPq), Brazil.

\section{References}

[1] WHO. Control of Chagas' disease, Report of a WHO expert committee. Geneva-WHO Technical Report Series, 811. 1991. pp. 27-31.

[2] Tibayrenc M, Ward P, Moya A, Ayala FJ. Natural populations of Trypanosoma cruzi the agent of Chagas' disease, have a complex multiclonal structure. Proc Natl Acad Sci USA 1986;83:6810-4.

[3] Laurent JP, Barnabé C, Quesney V, Noël S, Tibayrenc M. Impact of clonal evolution on the biological diversity of Trypanosoma cruzi. Parasitology 1997;114:213-8.

[4] Lana M, Pinto AS, Barnabé C, Quesney V, Noël S, Tibayrenc M. Trypanosoma cruzi: compared vectorial transmissibility of three major clonal genotypes by Triatoma infestans. Exp Parasitol 1998;90:20-25.

[5] Pinto AS, Lana M, Bastrenta B, et al. Compared vectorial transmissibility of pure and mixed clonal genotypes of Trypanosoma cruzi in Triatoma infestans. Parasitol Res 1998;90:20-25.

[6] Revollo S, Oury B, Laurent JP, et al. Trypanosoma cruzi: impact of clonal evolution of the parasite on its biological and medical properties. Exp Parasitol 1998;89:30-39.

[7] Brenière SF, Bosseno MF, Revollo S, Rivera MT, Carlier Y, Tibayrenc M. Direct identification of Trypanosoma cruzi natural clones in vectors and mammalian hosts by polymerase chain reaction amplification. Am J Trop Med Hyg 1992;46:335-41.

[8] Morel CM, Deane MP, Gonçalves AM. The complexity of Trypanosoma cruzi populations revealed by schizodeme analysis. Parasitol Today 1984;2:97-101.

[9] Brenière SF, Tibayrenc M, Antezana G, et al. Resultats préliminaires en faveur d'une relation faible ou inexistente entre les formes cliniques de la maladie de Chagas et les souches isoenzymatiques de Trypanosoma cruzi. CR Acad Sci Paris 1985;300:555-8.

[10] Tibayrenc M, Cariou ML, Solignac M, Dédet JP, Poch O, Desjeux P. New electrophoretic evidence of genetic variation and diploidy in Trypanosoma cruzi, the causative agent of Chagas' disease. Genetica 1985;67:223-30.

[11] Bosseno MF, Telleria J, Vargas F, Yaksic N, Noireau F, Morin A, Brenière SF. Trypanosoma cruzi: study of the distribution of two widespreaded clonal genotypes in Bolivian Triatoma infestans vectors shows a high frequence of mixed infections. Exp Parasitol 1996;83:275-82.

[12] Veas F, Cuny G, Brenière SF, Tibayrenc M. Subspecific kDNA probes for major clones of Trypanosoma cruzi. Acta Trop 1991;48:79-82.

[13] Barnabé C, Brisse S, Tibayrenc M. Population structure and genetic typing of Trypanosoma cruzi, the agent of Chagas' disease: a multilocus enzyme electrophoresis approach. Parasitology 2000;150:51326. 
[14] Tibayrenc M, Neubauer K, Guerrini F, Sarkeski D, Ayala FJ. Genetic characterization of six parasitic protozooa: parity between randomprimer DNA typing and multilocus enzyme electrophoresis. Proc Natl Acad Sci USA 1993;90:1335-9.

[15] Brisse S, Barnabé C, Tibayrenc M. Identification of six Trypanosoma cruzi phylogenetic lineages by random amplified polymorphic DNA and multilocus enzyme electrophoresis. Int J Parasitol 2000;30:3544.

[16] Avila HA, Sigman DS, Cohen LM, Millikan RC, Simpson L. Polymerase chain reaction amplification of Trypanosoma cruzi kinetoplast minicicle DNA isolated from whole blood lysates: diagnosis of chronic Chagas' disease. Mol Biochem Parasitol 1991;48:211-22.

[17] Britto C, Cardoso MA, Winker P, Morel CM. A simple protocol for the physical cleavage of Trypanosoma cruzi kinetoplast DNA present in blood samples and its use in polymerase chain reaction (PCR)based diagnosis of chronic Chagas' disease. Mem Inst Oswaldo Cruz 1993;88:171-2.

[18] Sturm NR, Degrave W, Morel C, Simpsom L. Sensitive detection and schizodeme classification of Trypanosoma cruzi cells by amplification of kinetoplast minicircle DNA sequences; use in diagnosis of Chagas' disease. Mol Biochem Parasitol 1989;33:205-14.

[19] Avila H, Gonçalves AM, Nehme NS, Morel CM, Simpson L. Schizodeme analysis of Trypanosoma cruzi stocks from South and Central America by analysis of PCR-amplified minicicle variable regions sequences. Mol Biochem Parasitol 1990;42:175-88.

[20] Tibayrenc M. Towards an integrated genetic epidemiology of parasitic protozoa and other pathogens. Annu Rev Genet 1999;33:44977. 